\title{
Maternal $\beta$-Hemolytic Streptococcal Pharyngeal Exposure and Colonization in Pregnancy
}

\author{
Giv Heidari-Bateni, ${ }^{1}$ Anoop K. Brar,, ${ }^{1}$ Matthew Hall,, ${ }^{2}$ Trupti Hathcock, ${ }^{1}$ Deirdre Epstein,, \\ Lisa S. Goessling, ${ }^{1}$ Madeleine W. Cunningham, ${ }^{3}$ and Pirooz Eghtesady ${ }^{1}$ \\ ${ }^{1}$ Division of Pediatric Cardiothoracic Surgery, Washington University School of Medicine, St. Louis, MO 63110, USA \\ ${ }^{2}$ Children's Hospital Association, 6803 West 64th Street, Overland Park, KS 66202, USA \\ ${ }^{3}$ Department of Microbiology and Immunology, The University of Oklahoma Health Sciences Center, 940 Stanton L. Young Boulevard, \\ BMSB 1053, Oklahoma City, OK 73104, USA
}

Correspondence should be addressed to Pirooz Eghtesady; eghtesadyp@wudosis.wustl.edu

Received 21 April 2014; Revised 23 July 2014; Accepted 24 July 2014; Published 20 August 2014

Academic Editor: Susan Cu-Uvin

\begin{abstract}
Copyright ( $\odot 2014$ Giv Heidari-Bateni et al. This is an open access article distributed under the Creative Commons Attribution License, which permits unrestricted use, distribution, and reproduction in any medium, provided the original work is properly cited.
\end{abstract}

Objectives. To report the pharyngeal colonization rate of $\beta$-hemolytic streptococci and changes in the value of antistreptolysin $\mathrm{O}$ (ASO) and anti-DNase B serology titers during pregnancy. Methods. Healthy pregnant women were recruited and blood was drawn in each trimester. The upper limit of normal (ULN) values for ASO and anti-DNase B was calculated for each trimester. Throat swabs were collected for culture and positive cultures were further assessed for the identification of serogroup of the isolated $\beta$ hemolytic streptococcus. Results. Out of a total of 126 pregnant women, $34.1 \%$ had positive throat cultures. Group $\mathrm{C}$ and group $\mathrm{G}$ strains were isolated in $18.2 \%$ of throat cultures while group $\mathrm{F}$ was detected in $13.5 \%$ of cases. The rate of colonization with GAS was $1.6 \%$. There was an overall drop in ASO titer during pregnancy while anti-DNase B titers remained relatively unchanged. ULN values of $164^{\mathrm{IU}}, 157^{\mathrm{IU}}$, and $156^{\mathrm{IU}}$ were calculated for ASO at the first, second, and third trimesters, respectively. Based on the ULN values, $28.6 \%$ of patients had recent streptococcal exposure. Conclusions. These results show that pregnant women act as a reservoir for spreading potentially immunogenic (groups $\mathrm{C}$ and $\mathrm{G}$ ) and disease producing (group F) virulent strains of streptococci.

\section{Introduction}

Pregnancy is associated with physiological changes in the respiratory system [1]. Prior studies have suggested that pregnant women, as a result of modulations in the immune system, are more susceptible to upper respiratory tract infections $[1,2]$. There are also concerns about the possible effect of respiratory infections during pregnancy on pregnancy outcomes [3-5]. Our group has previously suggested that maternal exposure to rheumatogenic groups of $\beta$-hemolytic streptococcus may play a role in pathogenesis of congenital heart disease in the unborn fetus [6], potentially mediated by streptococcal-induced anti-cardiac myosin antibodies [7]. There have been relatively few studies that have specifically studied $\beta$-hemolytic exposure during pregnancy. The point prevalence of $\beta$-hemolytic streptococcal pharyngeal colonization in the last trimester of pregnancy has been recently reported to be $8 \%$ in a small group of pregnant women in United Kingdom [8]. The pregnancy incidence rate, however, has not yet been studied and is anticipated to be much more than the reported point prevalence. For the general population, the two-year incidence rate has been reported to be $19.4 \%$ [9].

Although it is not well defined whether eradication of the asymptomatic carrier state is beneficial to the carrier hosts and their close contacts, it has been reported that the presence of carrier state might reduce the efficacy of penicillin G on eradicating group A streptococcal (GAS) pharyngitis $[10,11]$ and may be a source for both symptomatic or asymptomatic spread of GAS [12-14]. Furthermore, some strains of 
Lancefield groups C and G $\beta$-hemolytic streptococci (Streptococcus dysgalactiae subspecies equisimilis) possess common antigenic features with GAS [15-17] and can cause infections similar to GAS [8]. They are similarly attributed to induce rheumatogenic outcomes $[16,17]$.

The purpose of this study was to determine the rate of throat colonization and exposure to $\beta$-hemolytic streptococcus in pregnant women. We also defined the upper limit of normal (ULN) values of ASO and anti-DNase B titers in pregnant women.

\section{Materials and Methods}

2.1. Subjects. Pregnant subjects ( $>18$ years of age) were recruited in the first trimester of the pregnancy and participated in a total of 3 visits. The first visit was at $<18$ weeks of gestation, the second between 20 and 30 weeks, and the third between 30 and 38 weeks. Subjects who received a blood transfusion or immunoglobulin infusion within 6 months of the pregnancy were excluded from the study. Only data from those subjects who completed all 3 visits are reported here.

This study was initiated in Cincinnati and moved to St. Louis upon transition of the principal investigator to Washington University, St. Louis, MO. The Institutional Review Boards at the Washington University School of Medicine, St. Louis, Missouri, and Cincinnati Children's Hospital Medical Center, Cincinnati, Ohio, approved this study. Written informed consent was received prior to inclusion in the study.

2.2. Questionnaires and Streptococcus Culture. All subjects completed a short questionnaire (see supplemental file in Supplementary Material available online at http://dx.doi.org/10.1155/2014/639141) at the first visit. This questionnaire was used to determine the mothers' history of antecedent $\beta$-hemolytic streptococcal infection.

Two throat swabs were collected at each visit and were cultured and grouped in accordance with Centers for Disease Control and Prevention protocols for Lancefield streptococcal identification (methods as described at http://www.cdc.gov/ncidod/biotech/strep/protocols.htm. date accessed: $12 / 26 / 13$ ).

The incidence of $\beta$-hemolytic streptococcus throat colonization in pregnancy was defined as the number of subjects with at least one time positive throat culture divided by the total number of participants. The point prevalence of streptococcus throat colonization for each separate trimester was defined as the number of positive throat cultures divided by the total number of samples collected in that particular trimester. Carriers for $\beta$-hemolytic streptococcus were identified as subjects for whom the organism was isolated from at least two consecutive throat cultures while they were not symptomatic $[18,19]$, and there was no increase in ASO or anti-DNase B antibody titers [19].

2.3. ASO and Anti-DNase B Antibody Titers. Blood samples were collected from the subjects at each of the three visits to measure titers of ASO and anti-DNase B. Measurements of ASO and anti-DNase B titers were conducted by Quest
Diagnostics Incorporated using quantitative immunoturbidimetric assays (for ASO) and by either Wampole Streptonase kit or immunonephelometric technique (for anti-DNase B). In order to identify the prevalence of recent exposure to streptococcus in the pregnant population, we utilized UNL $20 \%$ values of ASO and anti-DNase B titers (measured by Wampole kit) as described in the data analysis section. For anti-DNase B titers measured by immunonephelometric technique, due to a limitation in sample size, the ULN value presented by the reference laboratory was used. Subjects with a titer more than the ULN 20\% value of either ASO or antiDNase B (or a titer more than laboratory reference for antiDNase B measured by the immunonephelometric technique) were considered as those with recent exposure to $\beta$-hemolytic streptococcus.

2.4. Data Analysis. Values of ASO and anti-DNase B titers were compared between trimesters using paired sample $t$ test. Changes in serology titers between pregnancy trimesters were measured for each subject; the median and the interquartile range for these changes within the study population were reported. Correlation between values of ASO and anti DNase-B antibodies was assessed for each pregnancy trimester.

Subjects were assigned into positive or negative groups based on throat culture results and the frequency of positive $\beta$-hemolytic streptococcus culture result in each trimester. The incidence of streptococcal colonization in pregnancy was measured as described earlier.

A ULN of $20 \%$ was considered as the 80 th percentile of cumulative frequencies of unexposed population titers $[20,21]$. Subjects with a titer more than the ULN $20 \%$ values were considered as those with recent exposure. In order to calculate the ULN value, subjects with evidence of infection with streptococcus from six months prior to blood draw were excluded as previously described $[20,21]$. For this aim, we excluded subjects who showed at least a two-time increase in the level of ASO or anti-DNase B during pregnancy. Also, we excluded subjects who were prescribed an antibiotic for a sore throat from the time point of 3 months before the pregnancy and the time of blood draw.

Demographic data and responses to the questionnaire were compared between exposed and unexposed individuals, as well as those with positive and negative cultures using Fisher's exact test and Mann-Whitney $U$ test to compare categorical and numerical data, respectively. A $P$ value $<0.05$ was considered as statistically significant when comparing the demographic data. The Bonferroni correction for $P$ value $(P=0.0025)$ was used to reduce the chances of obtaining false-positive results when comparing the questionnairebased data.

\section{Results}

A total of 126 subjects (from a total of 150 recruitments) presented for three visits between 18 and 38 weeks of pregnancy. Their demographic characteristics are shown in Table 1. A total of $7.9 \%$ of subjects were smokers. 
TABLE 1: Demographic characteristics of pregnant women.

\begin{tabular}{lc}
\hline Characteristics & $N(\%)$ \\
\hline Race & $103(81.7)$ \\
$\quad$ White & $18(14.3)$ \\
African American & $5(4.0)$ \\
$\quad$ Asian and others & $31(28-35)$ \\
Age (years) - median (IQR) & $64(59-78)$ \\
Weight at visit 1 (kg)—-median (IQR) & $24.1(21.3-28.6)$ \\
BMI at visit 1-median (IQR)
\end{tabular}

BMI: body mass index; IQR: interquartile range.

A comparison of the median change in ASO and antiDNase $\mathrm{B}$ titers between pregnancy trimesters revealed a decrease in ASO titers throughout pregnancy while antiDNase B titers did not change significantly between the second and third trimesters (Table 2). There was a positive correlation (but not strong) between values of ASO and antiDNase B in all three trimesters (Figure 1).

The ULN values of ASO and anti-DNase B antibody titers in each trimester are shown in Table 3. A total of 13 subjects were excluded before calculation of ULN as described in the methods of the study. The ULN value for ASO declined with progression of pregnancy, but this value for anti-DNase B stayed constant at the time points measured during pregnancy.

The chance of having a positive throat culture for $\beta$ hemolytic streptococci at the time points sampled in the 3 trimesters (including carrier state and asymptomatic infections) was $34.1 \%$. After excluding subjects with a previous history of tonsillectomy (32 patients), the incidence of having positive throat cultures was $40.4 \%$. With regard to the serogroup of $\beta$-hemolytic streptococci in the cultured isolates, group $\mathrm{C}$ and group $\mathrm{G}$ strains together comprised $18.2 \%$ of patients while group $\mathrm{F}$ was detected in $13.5 \%$ of cases. The rate of colonization with GAS was $1.6 \%$. Table 4 summarizes throat culture results.

The proportion of smokers was significantly greater in subjects with positive throat culture for $\beta$-hemolytic streptococci (at any stage of pregnancy) compared with those with negative culture results $(16.3 \%$ versus $3.6 \%, P=$ $0.013)$. Further, a greater proportion of subjects with positive cultures compared with negative cultures lived with a smoker (25.6\% versus $10.8 \%, P=0.032$ ). In addition, subjects with at least one positive culture at any time during pregnancy lived in households in which the median number of children was greater than in subjects with negative cultures (median: 3 [IQR: 2.5-5] versus 2 [IQR: 1-2], $P=0.001$ ).

Based on the ULN values, $28.6 \%$ of patients had recent streptococcal exposure. Subjects with laboratory evidence of recent strep exposure (defined as either ASO or anti-DNase $B$ antibody titers of equal to, or more than, the ULN value during pregnancy), showed a trend toward an association with asthma when compared with unexposed individuals (33.3\% versus $11.1 \%, P=0.003$ considering Bonferroni correction).

\section{Discussion}

In this study, we show for the first time, the rate of throat colonization with different groups of $\beta$-hemolytic streptococci among pregnant women in the US. Our data show a colonization rate of $34.1 \%$, most of which caused by non-GAS $\beta$-hemolytic streptococci (groups G, C, and F). Strömberg et al. reported this incidence rate to be $19.4 \%$ among nonpregnant population, in a period of 2 years which is much lower than the reported rate in pregnant population [9].

Asymptomatic pharyngeal carriage of $\beta$-hemolytic streptococcus is a state of colonization of pharyngeal mucosa without the presence of an infection and the serological response to streptococcal antigen $[18,19]$. As previously noted, some strains of groups $C$ and $\mathrm{G} \beta$-hemolytic streptococci cause infections similar to GAS [21] and can similarly induce rheumatogenic outcomes [16, 17]. A high colonization rate with these strains raises concerns about the possible emergence of drug-resistant rheumatogenic streptococci. Group F $\beta$-hemolytic streptococcus (S. milleri or S. anginosus group $\mathrm{F}$ ) is generally considered a normal resident of healthy adult pharyngeal mucosa [22]. Nevertheless, it has been associated with purulent infections, particularly in those with underlying diseases $[22,23]$. There were also reports of infections with S. milleri during pregnancy [24-26]. A high rate of colonization with group F streptococcus might also raise concerns about risk of $S$. milleri-induced infections in pregnant women, particularly in those with underlying medical conditions.

Our data show that the chance of colonization with $\beta$-hemolytic streptococcus is higher in pregnant women who are smokers and those with many children in the household compared with nonsmokers and/or households with few children. Evidence that smoking increases the rate of colonization with pathogenic respiratory agents has been previously reported $[27,28]$. It has been proposed that smoking could increase bacterial adherence by inducing injury and alteration in innate immune response in oropharyngeal epithelial cells $[29,30]$.

In this study, we also defined previously unpublished ULN values of ASO and anti-DNase B antibodies in pregnant population. The ULN value of ASO in adult population has been reported in a range between 177 and $250 \mathrm{iu} / \mathrm{mL}$ among different geographical regions [31, 32]. Reported rates are greater than the titers we detected in our study for pregnant women.

Our results show an overall drop in ASO levels over the course of pregnancy, with anti-DNase B titers remaining almost stable during the last two-thirds of pregnancy. Many studies have emphasized the importance of reporting antiDNase B titers to confirm recent streptococcal exposure $[33,34]$. In a study by Blyth and Robertson [33], to detect streptococcal exposure in subjects with poststreptococcal nonsuppurative disease, $95 \%$ sensitivity was reported for the application of a combination of ASO and anti-DNase B tests, suggesting an increase in either of these tests should be used to detect recent streptococcus exposure. In our study, we used either of these two tests to detect recent exposure. We also 
TABLE 2: Changes in ASO and anti-DNase B titers between pregnancy trimesters.

\begin{tabular}{|c|c|c|c|c|c|}
\hline & \multicolumn{3}{|c|}{ Mean (SD) } & \multirow{2}{*}{$\begin{array}{l}\text { Change T1 to T2 } \\
\text { median (IQR) }\end{array}$} & \multirow{2}{*}{$\begin{array}{l}\text { Change T2 to T3 } \\
\text { median (IQR) }\end{array}$} \\
\hline & 1st trimester & 2nd trimester & 3rd trimester & & \\
\hline ASO & $112.0(121.8)$ & $105.9(102.2)$ & $102.8(100.0)$ & $-8(-13,-1)^{\mathrm{a}}$ & $-5(-14,0)^{\mathrm{a}}$ \\
\hline \multicolumn{6}{|l|}{ Anti-DNase B } \\
\hline Wampole kit & $134.5(133.8)$ & $122.0(117.5)$ & $126.5(125.4)$ & $0(0,0)$ & $0(0,0)$ \\
\hline Nephelometer & $132.0(123.0)$ & $114.6(126.5)$ & $117.9(111.8)$ & $0(-45,0)^{\mathrm{b}}$ & $0(-2.5,1)$ \\
\hline
\end{tabular}

${ }^{\mathrm{a}} P<0.001$ (paired samples $t$-test).

${ }^{\mathrm{b}} P=0.035$ (paired samples $t$-test).

SD: standard deviation; IQR: interquartile range; ASO: antistreptolysin O.

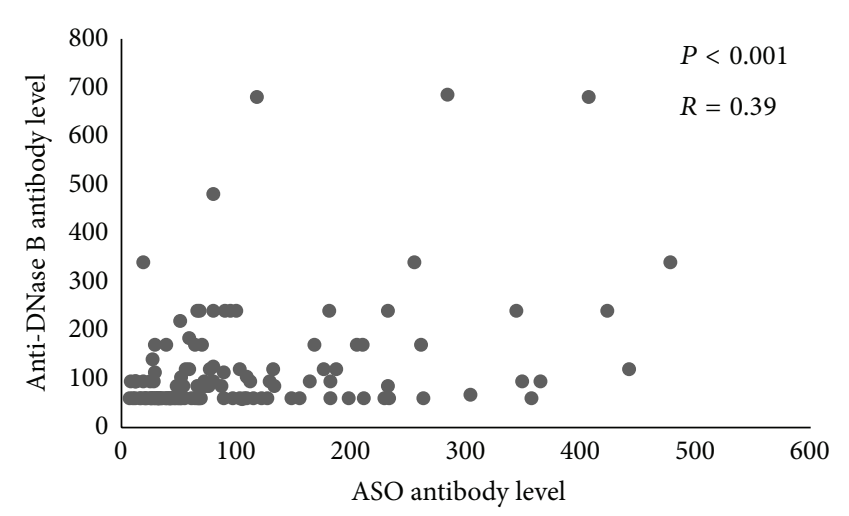

(a)

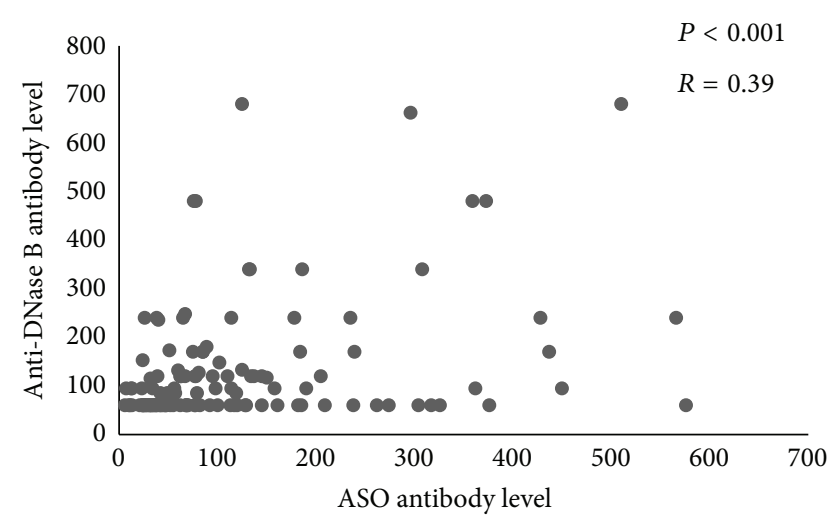

(b)

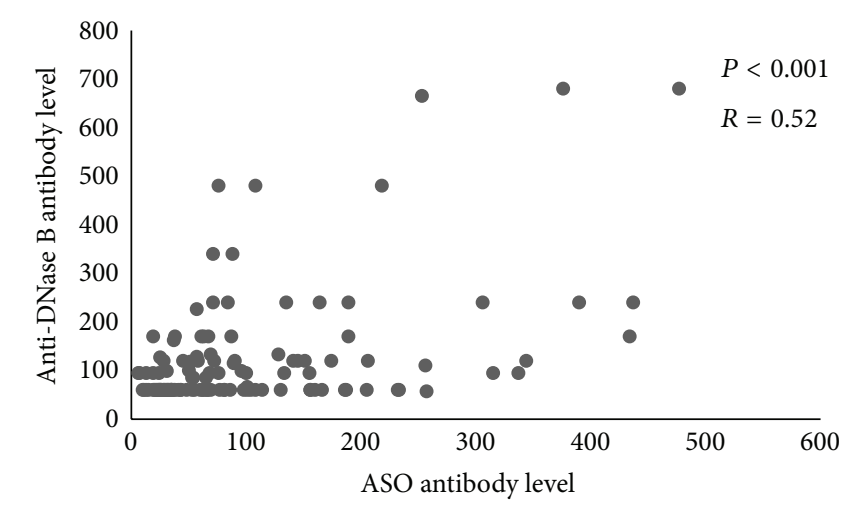

(c)

FIGURE 1: Correlation between ASO and anti-DNase B titers in pregnancy trimesters. Scatter plots showing correlation between values of ASO and anti-DNase-B antibodies in different pregnancy trimesters. Values less than laboratory lower limit for anti-DNase B detection were converted to the lower limit value of the assay. Correlation coefficients and Pearson correlation $P$ values are shown on the plots: (a) Trimester 1, (b) Trimester 2, and (c) Trimester 3.

TABLE 3: ULN values of the ASO and anti-DNase titers in pregnant women.

\begin{tabular}{lccc}
\hline & 1st trimester & 2nd trimester & 3rd trimester \\
\hline ASO & 164 & 157 & 156 \\
Anti-DNase B $^{\mathrm{a}}$ & 170 & 170 & 170 \\
\hline
\end{tabular}

${ }^{\mathrm{a}}$ Just considering anti-DNase B titers measured by Wampole kit. ULN: upper limit of normal; ASO: antistreptolysin O. showed a positive but not a strong linear correlation for levels of ASO and anti-DNase B; the correlation was found to be greater in the third trimester.

Pregnant women who have had recent exposure to $\beta$ hemolytic streptococcus were shown to have a trend toward having increased rates of asthma. A higher rate of GAS upper respiratory infection has been previously reported in asthmatic patients [35]. Whether the asthma is a risk 
TABLE 4: Frequency of throat isolation of $\beta$-hemolytic streptococci in each pregnancy trimester and in total.

\begin{tabular}{lcccc}
\hline Lancefield group & \multicolumn{2}{c}{ Point prevalence $N(\%)$} & Incidence $N(\%)$ \\
\hline A & 1st trimester & 2nd trimester & 3rd trimester & $2(1.6)$ \\
B & $1(0.8)$ & - & $2(1.8)$ & $3(2.4)$ \\
C & $2(1.6)$ & $6(4.8)$ & $7(5.6)$ & $15(11.9)$ \\
F & $5(4.0)$ & $9(7.1)$ & $8(6.4)$ & $17(13.5)$ \\
G & $5(4.0)$ & $2(1.6)$ & $6(4.8)$ & $9(6.3)$ \\
\hline Total & $5(4.0)$ & $19(15.1)^{\mathrm{a}}$ & $24(19.0)^{\mathrm{a}}$ & $43(34.1)^{\mathrm{a}}$ \\
\hline
\end{tabular}

${ }^{a}$ Some subjects had more than one group isolated during the pregnancy.

factor for acquiring infection with $\beta$-hemolytic streptococcus strains or repetitive exposure to streptococcal sore throat (pharyngitis) could result in hyperreactivity of airways has not yet been clarified and requires further studies.

One of the limitations for our study is that we applied ULN values for ASO and anti-DNase B titers to detect recent streptococcus exposure. A drawback for applying the serology-based concept of ULN for detection of recent streptococcus exposure is the probability that, in a minority of the cases, titers might not increase enough to pass the ULN value after a recent exposure. Checking both ASO and antiDNase B minimized this limitation, but it does not resolve it completely. Moreover, changes in ASO and anti-DNase B titer in pregnant women might be affected by serum volume changes during pregnancy. We were also unable to calculate, due to a limitation in sample size, an ULN 20\% value of anti-DNase B for samples measured by nephelometric assay. Further, we measured throat colonization three times during pregnancy which may not indicate the exact incidence rate of colonization with $\beta$-hemolytic streptococci during pregnancy. Also, it was not possible for us to differentiate the carrier state with the "state of recent asymptomatic infection" for subjects with just one time isolation of streptococcus from throat (isolated at either the first or the third trimester). Lastly, cautions must be taken into account when comparing culture results from various studies because the methodology used in isolating streptococcal species in different laboratories might potentially make a difference in culture results.

\section{Conclusion}

In summary, we report for the first time that pregnancy is associated with a high rate of colonization with streptococcal group $\mathrm{F}$ and also streptococcal groups $\mathrm{C}$ and $\mathrm{G}$ and this colonization is more prevalent among smokers. In addition, we provided for the first time a reference value for ASO and anti-DNase B titers for pregnant women in the United States. Results of this study suggest that one other variable that must be considered at the time of interpreting ASO and anti-DNase B titer results is the occurrence of pregnancy. Our work shows that pregnant women act as a reservoir for spreading potentially immunogenic (groups $\mathrm{C}$ and $\mathrm{G}$ ) and disease producing virulent (group F) strains of streptococci. How this colonization would affect the overall well-being of the mother, fetus, and households is an important question that warrants further investigations.

\section{Conflict of Interests}

The authors declare that there is no conflict of interests regarding the publication of this paper.

\section{Acknowledgment}

This work was supported by the National Institute of Health [Grant no. R501HL09863405].

\section{References}

[1] J. Harris and E. Sheiner, "Does an upper respiratory tract infection during pregnancy affect perinatal outcomes? A literature review," Current Infectious Disease Reports, vol.15, no. 2, pp. 143$147,2013$.

[2] R. E. Longman and T. R. Johnson, "Viral respiratory disease in pregnancy," Current Opinion in Obstetrics and Gynecology, vol. 19, no. 2, pp. 120-125, 2007.

[3] V. Laibl and J. Sheffield, "The management of respiratory infections during pregnancy," Immunology and Allergy Clinics of North America, vol. 26, no. 1, pp. 155-172, 2006.

[4] D. Getahun, C. V. Ananth, Y. Oyelese, M. R. Peltier, J. C. Smulian, and A. M. Vintzileos, "Acute and chronic respiratory diseases in pregnancy: associations with spontaneous premature rupture of membranes," Journal of Maternal-Fetal and Neonatal Medicine, vol. 20, no. 9, pp. 669-675, 2007.

[5] L. Stiller-Timor, A. Levy, G. Holcberg, and E. Sheiner, "Upper respiratory tract infection during pregnancy: is it associated with adverse perinatal outcome?" The American Journal of Perinatology, vol. 27, no. 8, pp. 619-624, 2010.

[6] C. R. Cole, K. E. Yutzey, A. K. Brar et al., "Congenital heart disease linked to maternal autoimmunity against cardiac myosin," The Journal of Immunology, vol. 192, no. 9, pp. 4074-9082, 2014.

[7] P. Eghtesady, "Hypoplastic left heart syndrome: rheumatic heart disease of the fetus?" Medical Hypotheses, vol. 66, no. 3, pp. 554$565,2006$.

[8] I. A. Hassan, T. S. Onon, D. Weston et al., "A quantitative descriptive study of the prevalence of carriage (colonisation) of haemolytic streptococci groups A, B, C and G in pregnancy," Journal of Obstetrics and Gynaecology, vol. 31, no. 3, pp. 207-209, 2011. 
[9] A. Strömberg, A. Schwan, and O. Cars, "Throat carrier rates of beta-hemolytic streptococci among healthy adults and children," Scandinavian Journal of Infectious Diseases, vol. 20, no. 4, pp. 411-417, 1988.

[10] E. L. Kaplan and D. R. Johnson, "Unexplained reduced microbiological efficacy of intramuscular benzathine penicillin $\mathrm{G}$ and of oral penicillin $\mathrm{V}$ in eradication of group A streptococci from children with acute pharyngitis," Pediatrics, vol. 108, no. 5, pp. 1180-1186, 2001.

[11] A. S. Gastanaduy, B. B. Huwe, E. L. Kaplan, C. McKay, and L. W. Wannamaker, "Failure of penicillin to eradicate group A streptococci during an outbreak of pharyngitis," The Lancet, vol. 2, no. 8193, pp. 498-501, 1980.

[12] A. G. Kuttner and E. Krumwiede, "Observations on the effect of streptococcal upper respiratory infections on rheumatic children: a three-year study," The Journal of Clinical Investigation, vol. 20, no. 3, pp. 273-287, 1941.

[13] W. E. James, G. F. Badger, and J. H. Dingle, "A study of illness in a group of Cleveland families. XIX: the epidemiology of the acquisition of group A streptococci and of associated illnesses.," The New England Journal of Medicine, vol. 262, pp. 687-694, 1960.

[14] L. Nguyen, D. Levi, A. Ferroni, P. Gehanno, and P. Berche, "Molecular epidemiology of Streptococcus pyogenes in an area where acute pharyngotonsillitis is endemic," Journal of Clinical Microbiology, vol. 35, no. 8, pp. 2111-2114, 1997.

[15] C. M. Brandt, G. Haase, N. Schnitzler, R. Zbinden, and R. Lütticken, "Characterization of blood culture isolates of Streptococcus dysgalactiae subsp. equisimilis possessing Lancefield's group A antigen," Journal of Clinical Microbiology, vol. 37, no. 12, pp. 4194-4197, 1999.

[16] A. Haidan, S. R. Talay, M. Rohde, K. S. Sriprakash, B. J. Currie, and G. S. Chhatwal, "Pharyngeal carriage of group C and group $\mathrm{G}$ streptococci and acute rheumatic fever in an Aboriginal population," The Lancet, vol. 356, no. 9236, pp. 1167-1169, 2000.

[17] K. Dinkla, D. P. Nitsche-Schmitz, V. Barroso et al., "Identification of a streptococcal octapeptide motif involved in acute rheumatic fever," Journal of Biological Chemistry, vol. 282, no. 26, pp. 18686-18693, 2007.

[18] J. Begovac, E. Bobinac, B. Benic et al., "Asymptomatic pharyngeal carriage of beta-haemolytic streptococci and streptococcal pharyngitis among patients at an urban hospital in Croatia," European Journal of Epidemiology, vol. 9, no. 4, pp. 405-410, 1993.

[19] E. L. Kaplan, "The group A streptococcal upper respiratory tract carrier state: an enigma," Journal of Pediatrics, vol. 97, no. 3, pp. 337-345, 1980.

[20] M. H. Danchin, J. B. Carlin, W. Devenish, T. M. Nolan, and J. R. Carapetis, "New normal ranges of antistreptolysin $\mathrm{O}$ and antideoxyribonuclease B titres for Australian children," Journal of Paediatrics and Child Health, vol. 41, no. 11, pp. 583-586, 2005.

[21] E. L. Kaplan, C. D. Rothermel, and D. R. Johnson, "Antistreptolysin $\mathrm{O}$ and anti-deoxyribonuclease B titers: normal values for children ages 2 to 12 in the United States," Pediatrics, vol. 101, no. 1, part 1, pp. 86-88, 1998.

[22] J. M. Whitworth, "Lancefield group F and related streptococci," Journal of Medical Microbiology, vol. 33, no. 3, pp. 135-151, 1990.

[23] F. Bert, M. Bariou-Lancelin, and N. Lambert-Zechovsky, "Clinical significance of bacteremia involving the "Streptococcus milleri” group: 51 cases and review," Clinical Infectious Diseases, vol. 27, no. 2, pp. 385-387, 1998.
[24] R. Lampen and G. Bearman, "Epidural abscess caused by Streptococcus milleri in a pregnant woman," BMC Infectious Diseases, vol. 5, article 100, 2005.

[25] A. P. MacGowan and P. B. Terry, "Streptococcus milleri and second trimester abortion," Journal of Clinical Pathology, vol. 40, no. 3, pp. 292-293, 1987.

[26] W. C. Hill, S. A. Armor, and G. R. G. Monif, "Perinatal bacteremia due to group F streptococci," The American Journal of Perinatology, vol. 9, no. 5-6, pp. 338-339, 1992.

[27] U. Jounio, A. Rantala, A. Bloigu et al., "Smoking status interacts with the association between mannose-binding lectin serum levels and gene polymorphism and the carriage of oropharyngeal bacteria," Human Immunology, vol. 71, no. 3, pp. 298-303, 2010.

[28] G. Piatti, T. Gazzola, and L. Allegra, "Bacterial adherence in smokers and non-smokers," Pharmacological Research, vol. 36, no. 6, pp. 481-484, 1997.

[29] B. W. M. Willemse, N. H. T. ten Hacken, B. Rutgers, D. S. Postma, and W. Timens, "Association of current smoking with airway inflammation in chronic obstructive pulmonary disease and asymptomatic smokers," Respiratory Research, vol. 6, article $38,2005$.

[30] M. Sopori, "Effects of cigarette smoke on the immune system," Nature Reviews Immunology, vol. 2, no. 5, pp. 372-377, 2002.

[31] A. C. Steer, S. Vidmar, R. Ritika et al., "Normal ranges of streptococcal antibody titers are similar whether streptococci are endemic to the setting or not," Clinical and Vaccine Immunology, vol. 16, no. 2, pp. 172-175, 2009.

[32] M. G. Karmarkar, V. Venugopal, L. Joshi, and R. Kamboj, "Evaluation \& revaluation of upper limits of normal values of anti-streptolysin O \& anti-deoxyribonuclease B in Mumbai," Indian Journal of Medical Research, Supplement, vol. 119, pp. 2628, 2004.

[33] C. C. Blyth and P. W. Robertson, "Anti-streptococcal antibodies in the diagnosis of acute and post-streptococcal disease: streptokinase versus streptolysin $\mathrm{O}$ and deoxyribonuclease B," Pathology, vol. 38, no. 2, pp. 152-156, 2006.

[34] S. Fujikawa and M. Okuni, "Asymptomatic infection by Streptococcus pyogenes in school children and diagnostic usefulness of antideoxyribonuclease B the determination of ADNase-B titers," Japanese Circulation Journal, vol. 43, pp. 417-418, 1979.

[35] D. Frey, R. Jacobson, G. Poland, X. Li, and Y. J. Juhn, "Assessment of the association between pediatric asthma and Streptococcus pyogenes upper respiratory infection," Allergy and Asthma Proceedings, vol. 30, no. 5, pp. 540-545, 2009. 


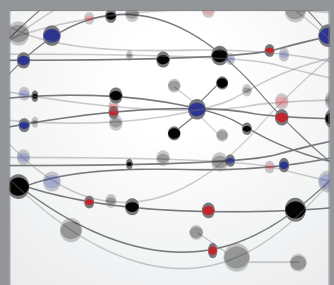

The Scientific World Journal
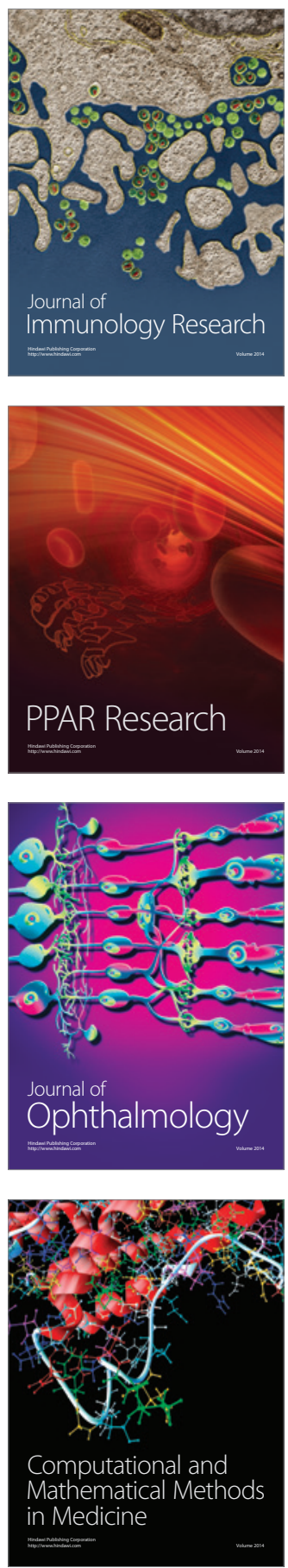

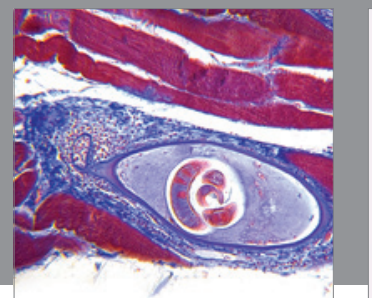

Gastroenterology

Research and Practice
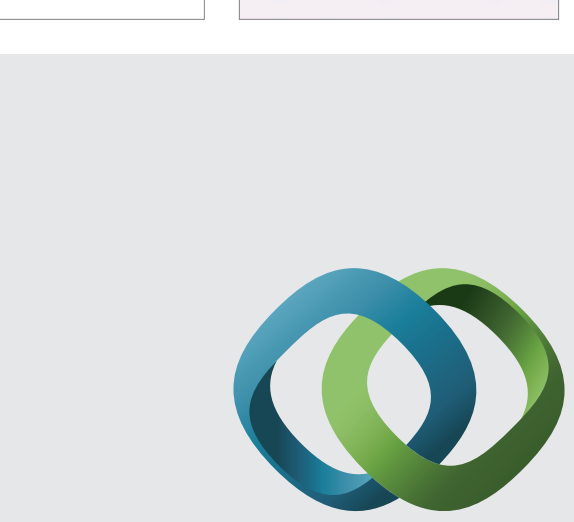

\section{Hindawi}

Submit your manuscripts at

http://www.hindawi.com
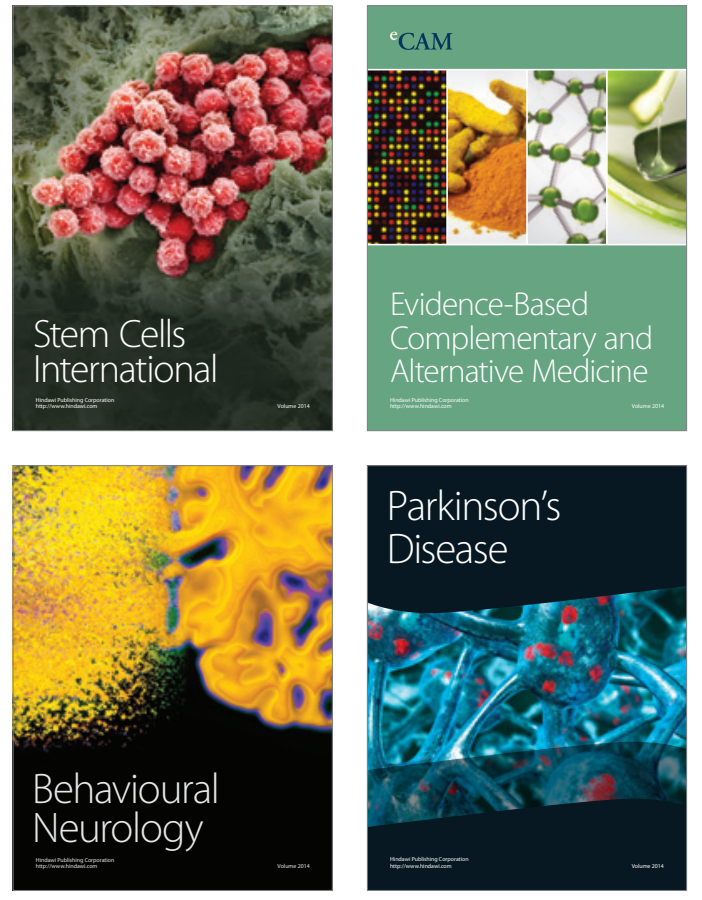
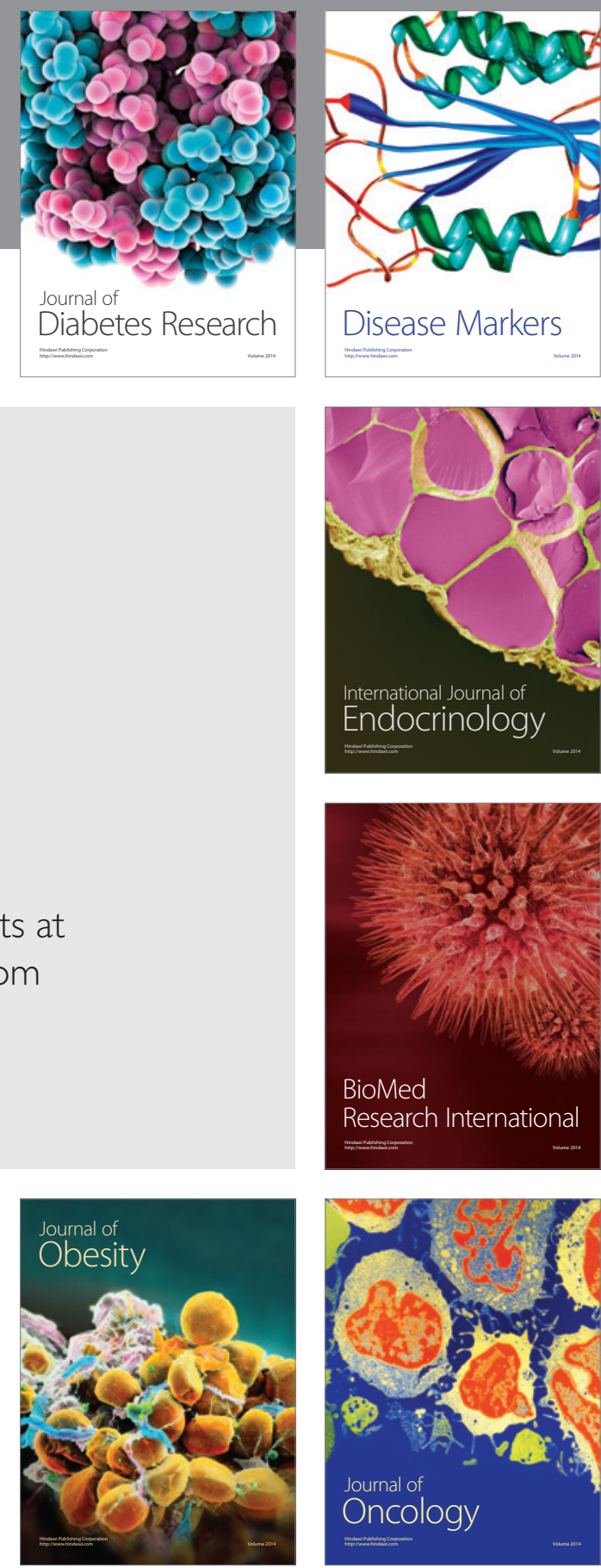

Disease Markers
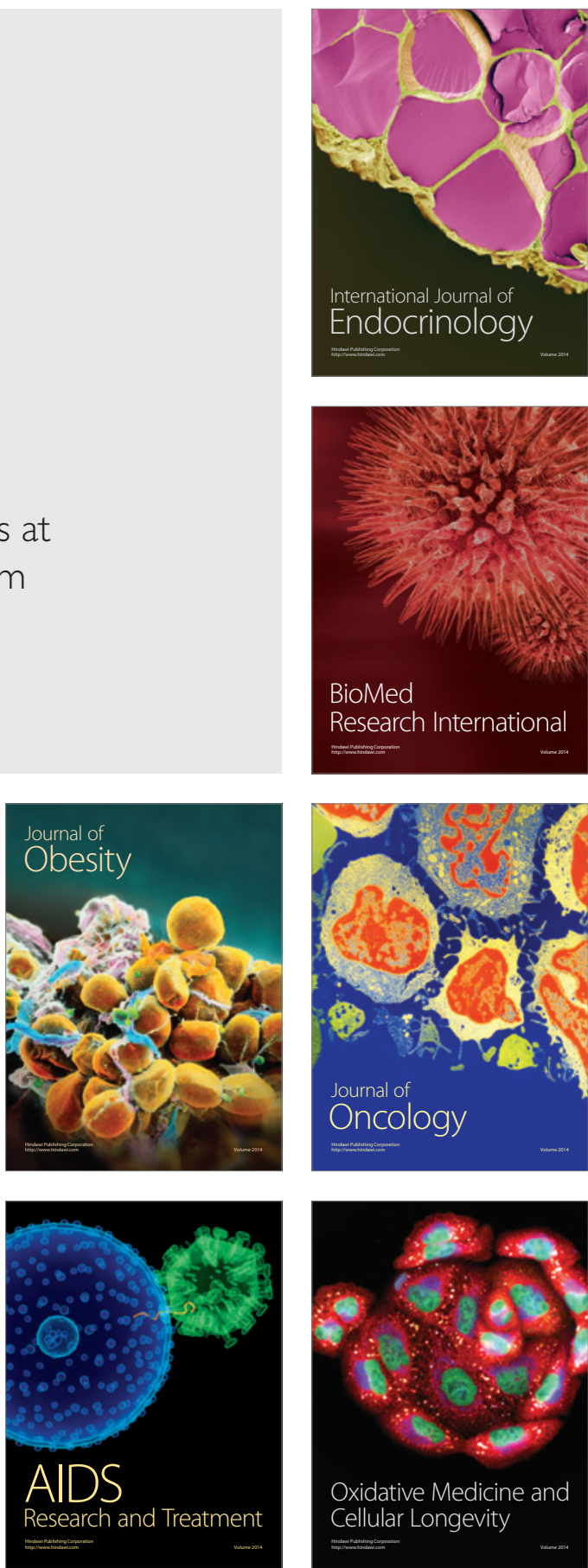